\title{
Apresentação: \\ Teoria e História da Arte: Abordagens Metodológicas
}

\author{
Comissão Organizadora do II EHA
}

\section{Agradecimentos:}

Secretaria de Eventos do IFCH

Casa do Lago

À Pró-reitoria de Extensão e ao Programa de Pós-Graduação em História do IFCH UNICAMP pelo apoio financeiro concedido. Aos professores do Marcos Tognon, Nelson Aguilar, Luiz Marques, Jorge Coli, Pedro Paulo Funari e Claudia Valladão de Mattos pela generosa ajuda com sugestões, dicas de organização e logística. Um especial agradecimento ao apoio irrestrito que os professores Marcos Tognon, Luciano Migliaccio e Luiz Marques nos deram. Ao professor Marcos Nobre por fazer viável a publicação no site da pós graduação do IFCH UNICAMP.

Aos professores de outras instituições que gentilmente vieram trocar e discutir novas perspectivas no II EHA: Maria Berbara (UERJ), Beatriz Ramos de Vasconcelos Coelho (UFMG), Luiz Alberto Freire (UFBA), Percival Tirapelli (UNESP), Maestro Henrique Liam (Secretaria de Cultura da Prefeitura de Campinas), Andrés Zarakin (Universidad de Buenos Aires), Gilson Rambelli (CEANS), Ana Paula Simioni (EACH USP) e Agnaldo Farias (USP).

À Secretaria de Eventos, em especial ao Irani e seu apoio técnico indispensável para que tudo desse certo nas apresentações das palestras, debates e comunicações. Ao pessoal da Informática que ajudou e elaborou a parte técnica desta publicação. A todos aqueles que auxiliaram, ajudaram, apoiaram, divulgaram e incentivaram a produção do II EHA e nossos maiores agradecimentos aos participantes, afinal sem vocês nada disso faria o menor sentido...

\section{Sobre o tema:}

O tema para o II EHA foi Teoria e História da Arte: abordagens metodológicas. Este evento foi organizado por uma comissão de alunos do doutorado e do mestrado em História da Arte do IFCH. Sua 
intenção inicial é dar continuidade ao esforço da primeira versão - também fruto do esforço do conjunto discente - e consolidar o espaço de comunicação entre os pesquisadores de todo o Brasil. Mais uma vez o objetivo foi prontamente alcançado e proporcionou a interação de estudiosos com diversas abordagens de pesquisa.

Seguindo o exemplo da edição anterior, nós continuamos abertos a todos os níveis de pesquisa, da iniciação científica aos estudos avançados de proeminentes historiadores e centros de pesquisa que vieram compartilhar suas reflexões com todos os presentes. Recebemos uma grande quantidade de propostas de comunicações e painéis, tanto que optamos por não mais termos duas sessões, mas quatro sessões ao mesmo tempo para podermos abrir oportunidade ao máximo de pessoas. Mesmo elaborando uma prévia seleção a partir dos resumos enviados o II EHA dobrou de tamanho em relação ao primeiro, o que acarretou para nós um estímulo para contribuirmos com a produção acadêmica em história da arte no Brasil. Teoria e História da Arte: abordagens metodológicas foi o tema escolhido pela comissão organizadora deste evento.

Esta temática abrange todos os campos e interesse e diálogo da História da Arte e anima discussões entre pesquisadores de todos os níveis. Nas palestras ministradas pelos professores Luiz Marques (IFCH UNICAMP), Luciano Migliaccio (FAU USP) e Maria Berbara (IA UERJ) as abordagens sobre a historiografia artística da tradição clássica foram amplamente debatidas. Na segunda sessão de palestras houve uma certa continuidade deste debate, pois o Barroco brasileiro foi tratado desde a abordagem da conservação até o universo da divulgação de estudos da arte brasileira, passando por revisões e discussões historiográficas detalhadas, feitas pelos professores Beatriz Ramos de Vasconcellos Coelho (CEIB UFMG), Luiz Alberto Freire (EBA UFBA) e Percival Tirapelli (IA UNESP). No segundo dia do evento contamos com os debates acerca do poder revolucionário contido nos objetos e nas apropriações que em diferentes momentos os artistas (re)elaboram na arte contemporânea.

O professor Nelson Aguilar (IFCH UNICAMP) com sua análise das 'Reciclagens revolucionárias de Lina Bo Bardi' e a apresentação do diálogo imagético tecido entre Aby Warburg e suas pranchas do 'Atlas Mnemosyne' com a apropriação deste formato pelo artista americano R. B. Kitaj e a influência deste sobre o artista brasileiro Wesley Duke Lee. Continaundo na mesma seara da arte contemporânea as palestras da tarde trataram do conceito de Conferência-Performática do maestro Henrique Liam. Ao mesmo tempo em que a professora Nancy Kaplan (IFCH UNICAMP) discorreu sobre Andrea Mantegna como eixo para tratar de problemas metodológicos acerca de Vasari e do Renascimento.

No último dia iniciamos os trabalhos com a apresentação dos pesquisadores Andrés Zarakin (Conicet e Universidad de Buenos Aires) e Gilson Rambelli (CEANS) sob a coordenação do professor Pedro 
Paulo Funari (IFCH UNICAMP). As palestras apresentadas abriram o universo da arqueologia com abordagens bastante específicas como a arqueologia do mundo contemporâneo e a arqueologia subaquática. Neste mesmo dia uma novidade, em relação à primeira edição foi colocada em prática: uma mesa-redonda com jovens pesquisadores que em comum possuem a dedicação aos estudos sobre o século XIX no Brasil.

A professora Ana Paula Simioni (EACH USP) coordenou e debateu sobre os desafios metodológicos da pesquisa em Arte do século XIX no Brasil, participaram desta mesa Elaine Dias (Doutora em História pelo IFCH e pós-doutoranda da FAU USP), Letícia Squeff (doutora pela FAU USP) e Renato Palumbo Dória (Doutor pela FAU USP e professor da UFU). Neste mesmo dia podemos contar com as palestras de encerramento dos professores Agnaldo Farias (FAU USP) que abordou os problemas da crítica de arte no panorama recente da arte brasileira e o professor Jorge Coli (IFCH UNICAMP) que apresentou uma fala inspirada sobre a especificidade da História da Arte.

Em todos os três dias do eventos houve comunicações que versaram sobre teoria e crítica de arte, arqueologia, as problemáticas de metodológicas das tradições clássicas, conservação, restauração e patrimônio, história da arquitetura, história do design, psicologia e arte, antropologia e arte, iconografia e iconologia, biografia de artistas, museus e museologia, arte e fotografia, novas tecnologias, ensino das artes, história da música e musicologia, colecionismo, literarua e arte, instituições e questões curatoriais, mecenato, imagem-texto, cinema, entre outros temas. E os painéis que ficaram expostos todos os dias na Casa do Lago.

\section{Programação}

\section{Primeiro dia - 27 de março de 2006}

o8hoomin Abertura das inscrições

08h45min Auditório 1: Abertura oficial do II Encontro de História da Arte e apresentação de pessoal de apoio. Coordenação: Prof. Dr. Marcos Tognon.

oghoomin Auditório 1: Palestras

Coordenação: Prof. Dr. Luiz Marques (Instituto de Filosofia e Ciências Humanas UNICAMP); Prof. Dr. Luciano Migliaccio (Faculdades de Arquitetura e Urbanismo USP);

Prof. ${ }^{\text {D }}$ Dr. ${ }^{\text {a }}$ Maria Berbara (Instituto de Artes UERJ).

10h3omin Café

11hoomin Mesas de Comunicação 1, 2, 3 e 4 
12hoomin Abertura da Exposição dos Painéis / Casa do Lago Almoço Livre 13h3omin Auditório 1: Palestras

Coordenação -Prof. Dr. Marcos Tognon; Profa. Emérita Beatriz Coelho (UFMG Centro de Estudos da Imaginária Brasileira); Prof. Dr. Luiz Alberto Freire (Escola de Belas Artes UFBA); Prof. Dr. Percival Tirapelli (Instituto de Artes UNESP).

15hoomin Café

15h3omin Mesas de Comunicação 5, 6, 7 e 8

16h3omin Mesas de Comunicação 9, 10, 11 e 12

18hoo Galeria de Arte da UNICAMP-Biblioteca Central Vernissage da exposição Acervos Artísticos da UNICAMP

\section{Segundo dia - 28 de março de 2006}

o8hoomin Inscrições

oghoomin Auditório 1: Palestras

Coordenação - Dra. Vanessa Beatriz Bortulucce; Prof. Dr. Nelson Aguilar (IFCH UNICAMP); Profa. Dra. Claudia Valladão de Mattos (Instituto de Artes UNICAMP) 10h3omin Café 11hoomin Mesas de Comunicação 13, 14, 15 e 16 12hoomin Almoço Livre 13h3omin Auditório 1: Palestras

Coordenação:Paula Vermeersch (IEL UNICAMP); Maestro Henrique Lian (Secretaria de Cultura da Prefeitura de Campinas); Profa. Dra. Nancy Kaplan (IFCH UNICAMP). 15hoomin Café 15h3omin Mesas de Comunicação 17, 18, 19 e 20 16h3omin Mesas de Comunicação 21, 22, 23 e 24 18hoomin Casa do Lago Lançamento dos Anais do I EHA Abertura da Exposição de fotografias Imagens e Viagens

\section{Terceiro dia - 29 de março de 2006}

o8hoomin Inscrições

oghoomin Auditório 1: Palestras

Coordenação -Prof. Dr. Pedro Paulo Funari (IFCH UNICAMP); Prof. Dr. Andrés Zarakin (Conicet e Universidad de Buenos Aires, Argentina); Profa. Dr. Gilson Rambelli (CEANS) 
10h3omin Café

11hoomin Mesas de Comunicação 25, 26, 27 e 28

12hoomin Almoço Livre

13h3omin Auditório 1: Mesa-Redonda: Debate acerca dos desafios metodológicos da pesquisa e da arte do século XIX no Brasil.

Coordenação: Helder Oliveira (IFCH UNICAMP); Debatedora -Profa. Dra. Ana Paula Simioni (EACH USP); Profa. Dra. Elaine Dias (FAU USP); Doutoranda Letícia Squeff (FAU USP); Prof. Dr. Renato Palumbo Dória (Universidade Bráz Cubas).

15h3omin Mesas de Comunicação 29, 30, 31 e 32

16h3omin Mesas de Comunicação 33, 34, 35 e 36

17h40min Auditório 1: Palestras de Encerramento

Coordenação: -Alexander Myioshi (IFCH UNICAMP); Prof. Dr. Agnaldo Farias (FAU USP);

Prof. Dr. Jorge Coli (IFCH UNICAMP).

\section{Comitê Organizador}

Ana Paula Camargo Lima

Christine De Carli

Helder Oliveira

Luciana Bicalho Piacenza

Marina Berriel

Patricia Sant'Anna

Apoio:

Pró-Reitoria de Extensão

Programa de Pós-Graduação em História do IFCH

FAEPEX

Secretaria de Eventos do IFCH

Casa do Lago 


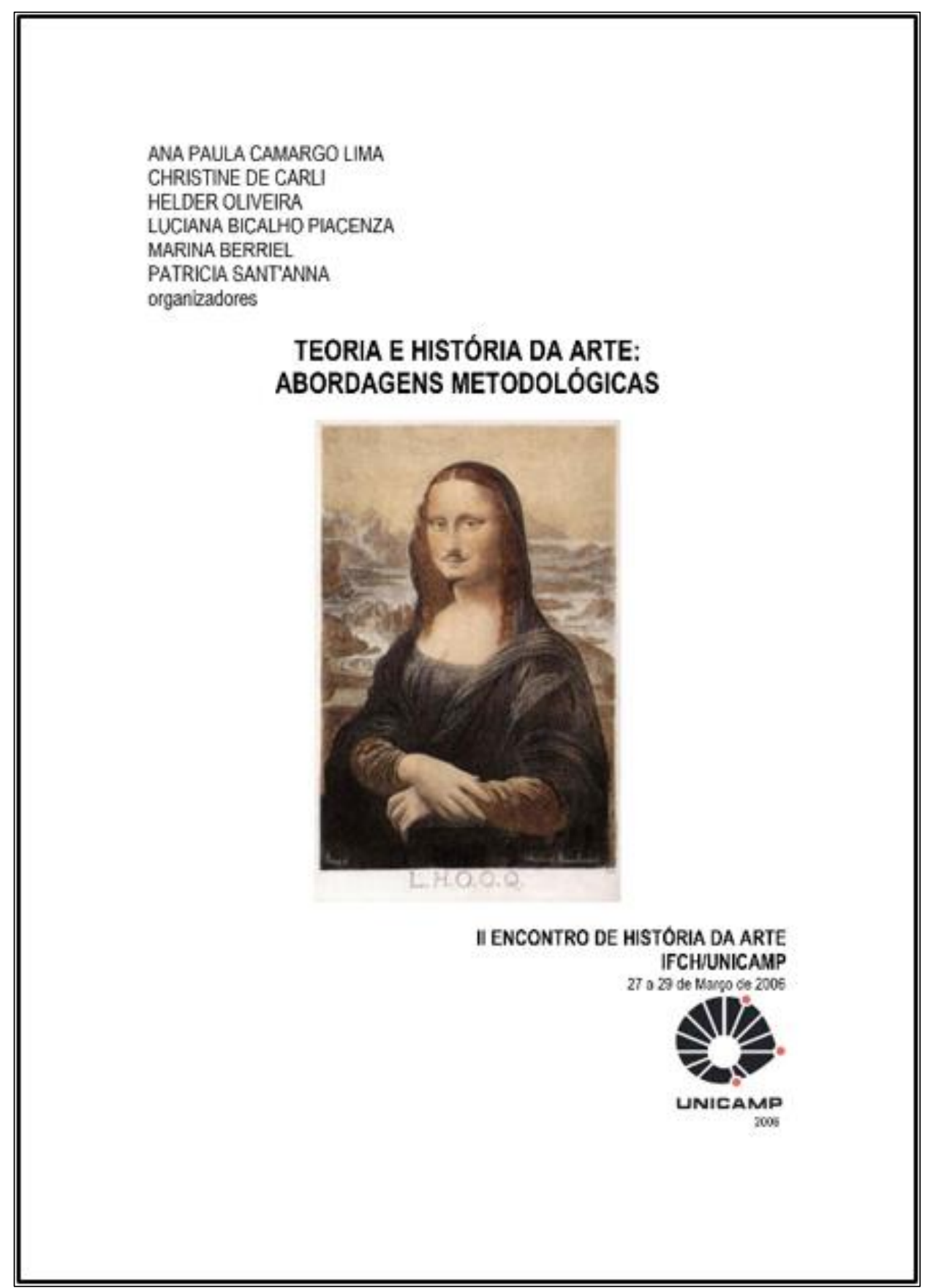

